\title{
Annular intimal tear of the aortic root misdiagnosed as aortic regurgitation
}

\author{
Chun Wang, MD, Zongyi Xiu, MD, and Tianxiang Gu, MD
}

\begin{abstract}
A 49-year-old man had a 6-month history of chest tightness and shortness of breath without any sudden chest pain. On examination, his blood pressure was $155 / 48 \mathrm{~mm} \mathrm{Hg}$, and a decrescendo sighlike diastolic murmur of grade $4 / 6$ could be heard at the Erb area. Water-hammer pulse, femoral pistol-shot sound, Duroziez murmur, and capillary pulsation were also detected. Transthoracic echocardiography showed a long, loose membrane structure $10 \mathrm{~mm}$ above the right coronary annulus (Figure 1) and prolapsing into the left ventricular outflow tract during the diastolic phase (Figure 2) with severe aortic regurgitation. The diastolic diameter of the left ventricle was $86 \mathrm{~mm}$, and the left ventricular ejection fraction was $69 \%$. The diameter of the aortic root was $37 \mathrm{~mm}$. The posteroanterior chest radiograph revealed an enlarged left ventricle.
\end{abstract}

Operation for aortic regurgitation was undertaken. During the operation, a midline incision of the chest was performed and antegrade cardioplegia was delivered through the aortic root; however, the heart could not be arrested. When the aortic root was cut open, the annular intimal tear, which was prolapsed into the outflow tract of the left

From the Department of Cardiac Surgery, The First Affiliated Hospital of China Medical University, Shenyang, China.

Disclosures: Authors have nothing to disclose with regard to commercial support.

Received for publication May 1, 2011; accepted for publication May 5, 2011; available ahead of print June 13, 2011.

Address for reprints: Tianxiang Gu, MD, Department of Cardiac Surgery, The First Affiliated Hospital of China Medical University, No. 155 N Nanjing St, Heping District, Shenyang, China, 110001 (E-mail: cmugtx@sina.com).

J Thorac Cardiovasc Surg 2011;142:706-7

$0022-5223 / \$ 36.00$

Copyright (c) 2011 by The American Association for Thoracic Surgery

doi:10.1016/j.jtcvs.2011.05.003

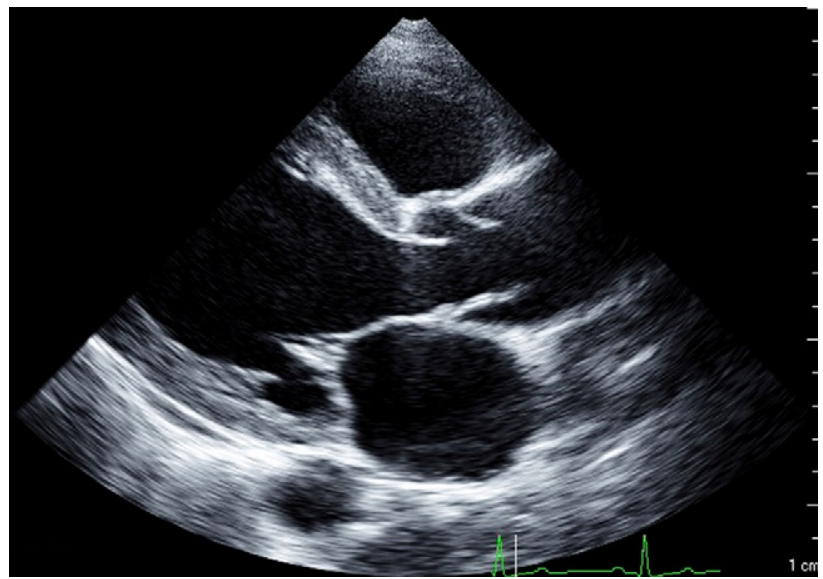

FIGURE 1. Transthoracic echocardiogram showing a long, loose membranous structure $10 \mathrm{~mm}$ above the right coronary annulus.

ventricle, could be seen above the aortic annulus (Figures 3 and 4). The antegrade cardioplegia was then delivered through the coronary ostia. After repair of the tear in the aortic root with felt (Figure 5), the regurgitation of the aortic valve disappeared.

The entity described here was a rare variant of aortic dissection that was characterized by an annular intimal tear without progression and separation of the medial layers, resulting in extensive undermining of the intimal layers. We are concerned that current imaging techniques may be inadequate for diagnosis of this type of aortic dissection, as in this case. The inability to identify this type of dissection preoperatively may result in poor prognosis.

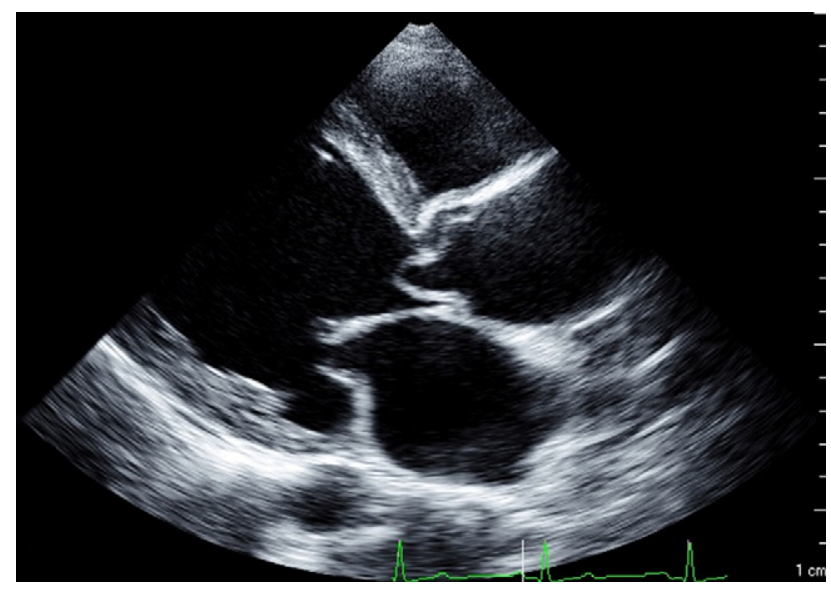

FIGURE 2. Transthoracic echocardiogram showing prolapse of a membranous structure into the left ventricular outflow tract during the diastolic phase. 


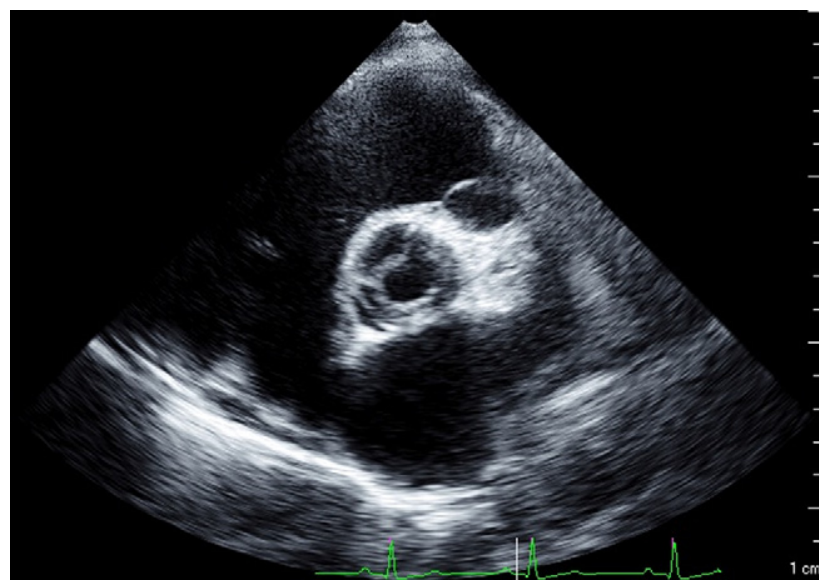

FIGURE 3. Preoperative transthoracic echocardiogram of the annular intimal tear in short-axis view of the aorta.

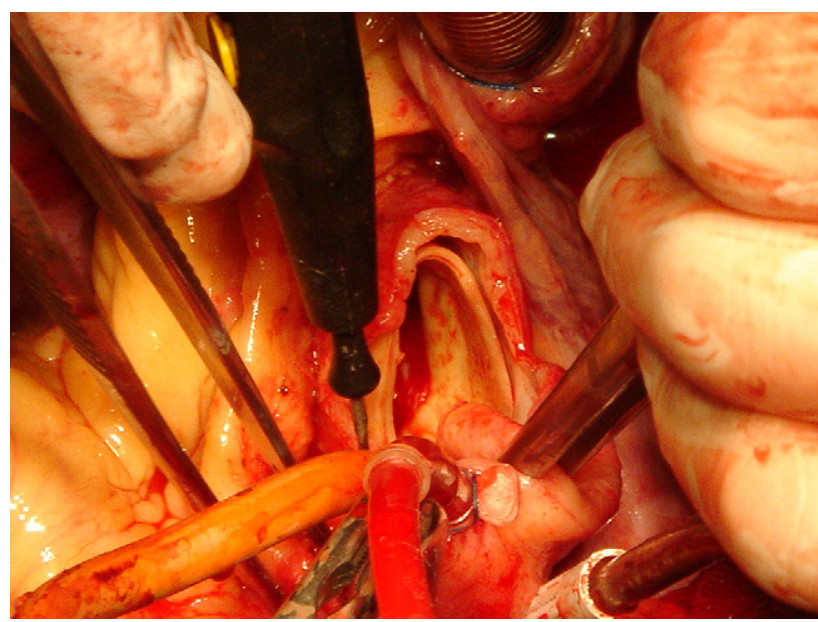

FIGURE 4. Intraoperative photograph showing opened aortic root to reveal the annular intimal tear prolapsed into the outflow tract of the left ventricle above the aortic annulus. The figure shows what has been revealed by Figure 3 before surgery.

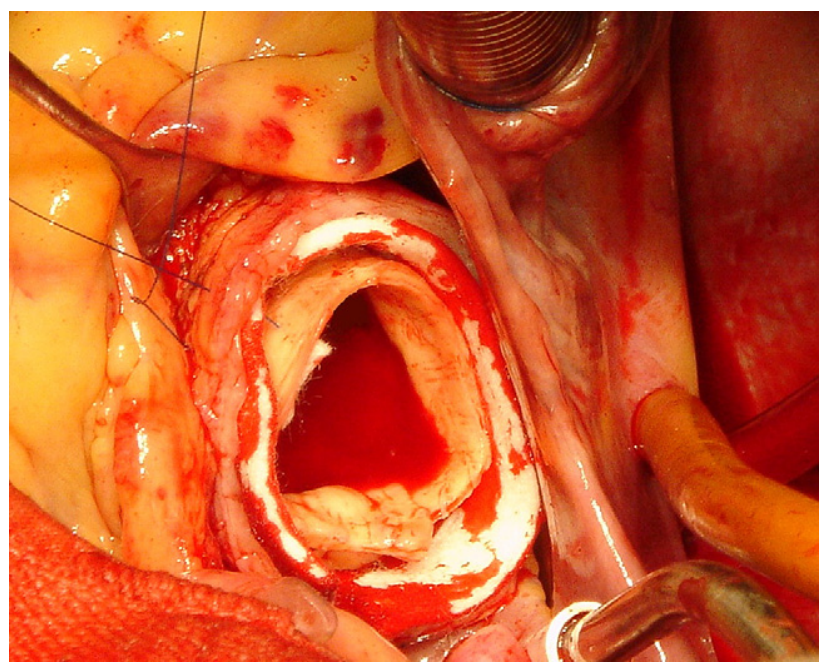

FIGURE 5. Intraoperative photograph showing repair with felt of the tear in the aortic root. This repair corrected the aortic regurgitation. 